\title{
Derivation of Field Equations in Space with the Geometric Structure Generated by Metric and Torsion
}

\author{
Nikolay Yaremenko \\ Yu. A. Mitropolsky Department, International Mathematical Center. JO Mitropolsky, Kiev 01601, Ukraine \\ Correspondence should be addressed to Nikolay Yaremenko; math.kiev@gmail.com
}

Received 21 August 2014; Revised 29 October 2014; Accepted 29 October 2014; Published 11 December 2014

Academic Editor: Kazuharu Bamba

Copyright (C) 2014 Nikolay Yaremenko. This is an open access article distributed under the Creative Commons Attribution License, which permits unrestricted use, distribution, and reproduction in any medium, provided the original work is properly cited.

\begin{abstract}
This paper is devoted to the derivation of field equations in space with the geometric structure generated by metric and torsion tensors. We also study the geometry of the space generated jointly and agreed on by the metric tensor and the torsion tensor. We showed that in such space the structure of the curvature tensor has special features and for this tensor we obtained analog Ricci-Jacobi identity and evaluated the gap that occurs at the transition from the original to the image and vice versa, in the case of infinitely small contours. We have researched the geodesic lines equation. We introduce the tensor $\pi_{\alpha \beta}$ which is similar to the second fundamental tensor of hypersurfaces $Y^{n-1}$, but the structure of this tensor is substantially different from the case of Riemannian spaces with zero torsion. Then we obtained formulas which characterize the change of vectors in accompanying basis relative to this basis itself. Taking into considerations our results about the structure of such space we derived from the variation principle the general field equations (electromagnetic and gravitational).
\end{abstract}

\section{Introduction}

In this paper we study the properties of the geometry of the space generated jointly and agreed on by the metric tensor and the torsion tensor, so we investigate the spaces with connection in the presence of the metric tensor. We obtained some results on the structure of the curvature tensor and considered the construction of geodesic lines and an estimate of the gap that occurs when traversing the contour of a parallelogram in these spaces.

The investigation of properties of metric spaces and affine connection spaces began approximately at the beginning of the 20th century $[1,2]$ and continued to develop so far [2-16].

The importance of this kind of research is on the one hand due to the internal logic of mathematical science bases itself $[1,2,9,13]$ and on the other hand to applications to problems in physics, analytical and theoretical mechanics $[3,12]$, the theory of relativity $[7,14-16]$, and continuum mechanics and cosmology [10]. Fairly well studied Riemann spaces [9], because of the wealth of geometric properties and less explored space with affine connection [5], are not sufficiently considered the most interesting geometry, which is obtained by combining geometry and affine connection generated by the metric tensor, and this is the subject of this work. From the theory of spaces with affine connection it is known that parallel displacement vector depends on pathways; that is, if the vector is parallel transported at the given contour with it return to the starting point, we obtain the other vector than the original (the gap appears). In the spaces, which are studied in this work, not only do they hold a similar statement, but also there are new properties of this gap.

The principle of least action (more correctly, the principle of stationary action) is the basic variational principle of particle and continuum systems. Let the starting point be the action, denoted as $S$, of a physical system. It is defined as the integral of the Lagrangian $L$ between two instants of time, a functional of the $n$ generalized coordinates $q$ which define the configuration of the system:

$$
S(q(t))=\int_{t_{1}}^{t_{2}} L(q(t), \dot{q}(t)) d t
$$

where the dot denotes the time derivative and $t$ is time. Mathematically the principle is $\delta S=0$, where $\delta$ means a variation. 
In applications the statement and definition of action are taken together:

$$
\delta \int_{t_{1}}^{t_{2}} L(q(t), \dot{q}(t)) d t=0 .
$$

The action and Lagrangian both contain the dynamics of the system for all times. The term "path" simply refers to a curve traced out by the system in terms of the coordinates in the configuration space, that is, the curve $q(t)$, parameterized by time. On the other hand, a Finsler manifold is a differentiable manifold together with the structure of an intrinsic quasimetric space in which the length of any rectifiable curve $\tau:[a, b] \rightarrow M$ is given by the length functional

$$
S(\tau(t))=\int_{t_{1}}^{t_{2}} F(\tau(t), \dot{\tau}(t)) d t,
$$

where $F(x, \cdot)$ is a Minkowski norm on each tangent space. It is obvious from these definitions that there is a connection between these two concepts, which can be realized by Hamiltonian formalism. Thus any Riemannian space can be regarded as Finsler manifold with the length functional: $F^{2}=$ $g_{i j}(x) d x^{i} d x^{j}$ and so the geodesics of a Finsler manifold are geodesics of Riemannian space.

The geodesics of the space that are being studied in our work (with the geometric structure generated by metric $g_{i j}(x)$ and torsion $S_{i j}^{k}(x)$ tensors) are different from geodesics of corresponding Riemann space (with $g_{i j}(x)$ ) and so of geodesics Finsler manifold (with $F^{2}=g_{i j}(x) d x^{i} d x^{j}$ ).

These spaces retain all the properties of geometry of an affine space but important features associated with the presence of the metric appear; the structure of the curvature tensor has a specific characteristics, as well as an opportunity to assess the gap that occurs at the transportation from the original to the image and conversely in the case of infinitely small contours.

The main objective of this work-the study of the geometric properties of the space that is generated by metric $g_{i j}(x)$ and torsion $S_{i j}^{k}(x)$ tensors; obtain the field equations from variation principle in such spaces.

We remind that according to Albert Einstein proposal: the free falling gravitating massive bodies follow geodesic line. If we postulate this proposal, we can obtain some results of Newton theory as a consequence. We have another important assumption of Albert Einstein that the geodesic equation of motion can be derived from the field equations for empty space.

Since we believe that gravitational and electromagnetic fields are determined geometric structure of empty space (torsion and curvature), it is interesting to have example about geometric sense of torsion.

Now, we discuss one example about geometric sense of torsion. We consider the surface $S$. At point $A$ on $S$ construct a tangent plane $P$. We choose an arbitrary infinitesimal square $A B C D$ in the plane $P$ with vertex $A$. From point $A$ on the surface $S$ will draw the geodesic in the direction of $A B$. We pass along the distance corresponding parameter equal to the length of $A B$ and get to point $B^{\prime}$. Similarly, from $A$ on $S$, draw geodesic towards $A D$ and get into $D^{\prime}$. We perform a parallel transportation of vector $A D$ to point $B^{\prime}$ along the geodesic $A B^{\prime}$ and draw geodesic $B^{\prime}$ along the transportation of this vector; we reach the point $C^{\prime}$. Similarly, the vector $A B$ will move parallel along the $A D^{\prime}$ and along the transported vector from $D^{\prime}$ draw a geodesic to get to $C^{\prime \prime}$. If torsion is zero, then $C^{\prime}=C^{\prime \prime}$, and geodesic square up to small higher order will be closed, otherwise not. In our case, due to the presence of the metric can calculate the length of the gap, more precisely we can estimate the length of this gap.

These considerations are true only up to the second order relative to the length of square side. If we want more strict result we must consider the component of curvature tensor. Next this example is true only when length of square side tends to zero that is, remains very small in other words in general it is a local property.

We go to the discussion of physical interpretation of this example. The physical properties of the space-time (more just space) are defined by the presence of matter (electromagnetic fields and mass) in this space and from the viewpoint of mathematics are described by the geometrical structure of space (torsion and metric tensors). The empty space (without matter) corresponds to the geometric structure of Euclidean space (torsion tensor and curvature tensor are identically equal to zero). Similarly gravitation (mass and without electromagnetic fields) corresponds to the geometric structure of Riemannian space (torsion tensor is identically equal to zero). And similarly the electromagnetism (electromagnetic fields and without mass) corresponds to the geometric structure of affine connection space (curvature tensor is identically equal to zero). In the last two cases the result is conditional (not strict) because the matter division by the mass and field is conditional.

To describe the internal geometry of the subspace is sufficient to define two tensors, the metric tensor $g_{i k}$ and the torsion tensor $S_{i k}^{m}$; using these tensors one can build connection of the space and all the geometry of the space.

If we consider the space of hypersurface $Y^{n-1}$ as a subspace then for a complete description of the geometry it is necessary and sufficient to define the tensors $a_{\alpha \beta}$ and $T_{\alpha \beta}^{\gamma}$. If we consider the hypersurface $Y^{n-1}$ as a subspace that embedded in a space $Y^{n}$ then it is not sufficient to know tensors $a_{\alpha \beta}$ and $T_{\alpha \beta}^{\gamma}$ for identifying the space $Y^{n}$ (for obtaining the metric tensor $g_{i k}$ and the torsion tensor $S_{i k}^{m}$ ); to describe the geometry of the ambient space it is necessary and sufficient to specify exactly how the hypersurface $Y^{n-1}$ is embedded in it that is, specify the embedment; it can be done in various ways. We assume that Jacobi matrix is known, so the way of embedment hypersurface $Y^{n-1}$ in $Y^{n}$ is determined (also the space can be recovered by determination of normal for the hypersurface $Y^{n-1}$ ).

We consider the following method of constructing spacetime: the space is constructed on the basis of manifolds by determining these manifolds metric tensor and torsion tensor. Metric and torsion tensors are calculated from the differential equations of the field. Hence torsion as the curvature 
arises from the physical features of the distribution of matter in space-time. Roughly, the same way as the masses leads to curvature space-time, electromagnetism leads to appearance of torsion. But on the other hand from the mathematical point of view if we assume that the space-time embedded in Euclidean space of higher dimension then the appearance of torsion can be explained by violation of the smoothness embedding. Therefore, we can conditionally determine the torsion and curvature by violation of smoothness regardless of the dimension and embedment.

The aim of our paper is to study the property of metric space with torsion and obtain analog of Einstein-Hilbert equation at such space.

This paper is organized as follows. In Section 2 some general properties of structure of a metric space with torsion are discussed. In Section 3 we present the study of geodesics in the space with torsion. These results can be used in "geodesic principle." In Section 4 the theory of hypersurfaces in the space with torsion is dedicated. In Section 5 we obtained some interesting relationships which is used in Section 6. In Section 6 we derive analog of Einstein-Hilbert (for electromagnetic and gravitational fields) in case of a metric space with torsion.

The main natural assumption that is used below that a scalar product of two any vectors that is transporting parallel along an arbitrary path does not change of the 20th century $[2,8,11-13]$, and continues to develop so far $[3-7,14-22]$.

\section{Structure of a Metric Space with Torsion}

There are many ways to represent the physical fourdimensional space, where events of our reality are occurring. From a mathematical point of view there are two possible conceptions of space geometry, which might be identified with the physical space.

The first scheme is a generalization of Euclidean geometry, the geometry of the Riemannian metric, that is, $n$ dimensional manifold equipped with a field twice covariant symmetric metric tensor which is non-degenerate $g_{i k}(M)$, where Det $\left|g_{i k}\right| \neq 0$ and $g_{i k}=g_{k i}$. Note that the metric tensor is chosen arbitrarily, but in addition to conditions laid above we demand that the manifold was sufficiently smooth.

This definition can be rewritten as follows: invariant differential quadratic form $g_{i k} d x^{i} d x^{k}$ determined on the manifold and satisfying the conditions Det $\left|g_{i k}\right| \neq 0 ; g_{i k}=g_{k i}$ defines the geometry of Riemann.

As a consequence of the invariance of the form

$$
d s^{2}=g_{i k} d x^{i} d x^{k}
$$

we find that the coefficients $g_{i k}$ are forming a tensor field.

In this model for the arc length of the curve $x^{i}=x^{i}(t)$, $t \in[a, b] \subset R$, is given by integral

$$
\begin{aligned}
s & =\int_{a}^{b} \sqrt{g_{i k} d x^{i} d x^{k}} \\
& =\int_{a}^{b} \sqrt{g_{i k}\left(x^{1}(t), \ldots, x^{n}(t)\right) \frac{d x^{i}(t)}{d t} \frac{d x^{k}(t)}{d t}} d t .
\end{aligned}
$$

The second scheme is a generalization of affine geometry, the geometry of affine connection $\Gamma_{j k}^{i}(M)$ that is based on $n$ dimensional manifold.

The connection $\Gamma_{j k}^{i}(M)$ is a geometric object on a manifold and is subjected to the law of the transformation from one coordinate system $x^{i}$ to another $x^{i^{i}}$ in the form of

$$
\Gamma_{j^{\prime} k^{\prime}}^{i^{\prime}}=\Gamma_{j k}^{i} \frac{\partial x^{i^{\prime}}}{\partial x^{i}} \frac{\partial x^{j}}{\partial x^{j^{\prime}}} \frac{\partial x^{k}}{\partial x^{k^{\prime}}}+\frac{\partial^{2} x^{i}}{\partial x^{j^{\prime}} \partial x^{k^{\prime}}} \frac{\partial x^{i^{\prime}}}{\partial x^{i}},
$$

where the functions $\Gamma_{j k}^{i}$ are sufficiently smooth.

Let along the curve $x^{i}=x^{i}(t), t \in[a, b] \subset R$ is given tensor field $A^{i}=A^{i}(t)$, if for each infinitesimal displacement tensor $d A^{i}(t)$ coordinates is changing the law:

$$
d A^{i}=-\Gamma_{j k}^{i} A^{j} d x^{k}
$$

then we say that the tensor $A^{i}$ is transported parallel to the curve $t$.

Depending on the physical investigated problem one or another geometric model is choosing, but as the internal logic and common sense requires that in the physical world, these two models coexist together and complement each other. There is well-known result that in an arbitrary Riemannian space can always construct a connection $\Gamma_{j k}^{i}(M)$. An interesting question is the uniqueness of such a construction. In general, such a construction $\Gamma_{j k}^{i}$ is not unique, but completely natural (in terms of mathematics and physics to a greater extent), there is the requirement that whenever two vectors $A^{i}$ and $B^{i}$ simultaneous parallel transported along a path (due to the presence of the connection the transportation is defined), their scalar product does not change (the scalar product is defined by metric). Mathematically, this can be written as the vanishing differential:

$$
d\left(g_{i k} A^{i} B^{k}\right)=0
$$

If the requested coefficients $\Gamma_{j k}^{i}$ are symmetric, namely, $\Gamma_{j k}^{i}=\Gamma_{k j}^{i}$, then the connectivity is uniquely defined using a metric.

Always below we would not require the symmetry of connection. And so if the metric $g_{i k}$ is defined, then a geometric object $\Gamma_{j k}^{i}$ subject to certain requirements, but still there is some arbitrariness in the choice of connectedness of the space; namely, we need to define a torsion tensor

$$
S_{j k}^{i} \equiv \Gamma_{j k}^{i}-\Gamma_{k j}^{i}
$$

then the geometric object $\Gamma_{j k}^{i}$ that generated the connection is uniquely determined.

Theorem 1. Suppose that a Riemannian space with the metric $g_{i k}$ and in this space is given torsion tensor $S_{j k}^{i}$-antisymmetric. If demand $d\left(g_{i k} A^{i} B^{k}\right)=0$ for arbitrary $A^{i}$ and $B^{k}$ then the connection (geometric object that defines it) $\Gamma_{j k}^{i}$ is uniquely defined. 
Proof. It is pretty easy to see the truth of this statement itself, but for further more importantly those symbols and values that relate to the nature of the entered values.

We rewrite $d\left(g_{i k} A^{i} B^{k}\right)=0$, as $\left(g_{i k, l}-g_{m k} \Gamma_{i l}^{m}-\right.$ $\left.g_{i m} \Gamma_{k l}^{m}\right) A^{i} B^{k} d x^{l}=0$ due to the fact that $d A^{i}=-\Gamma_{p l}^{i} A^{p} d x^{l}$, where $\Gamma_{i l}^{m}$, unknown coefficients of connection are a geometric object and $d x^{l}$ are the differentials of coordinates of a point under infinitesimal displacement along the path; $g_{i k, l} \equiv$ $\left(\partial / \partial x^{l}\right) g_{i k}$. Since, $A^{i}, B^{k}, d x^{l}$ are arbitrary, the equalities must be identity relative to $A^{i}, B^{k}, d x^{l}$. By circular permutation we obtain the system of equations:

$$
\begin{aligned}
& g_{i k, l}=g_{m k} \Gamma_{i l}^{m}+g_{i m} \Gamma_{k l}^{m}, \\
& g_{l i, k}=g_{m i} \Gamma_{l k}^{m}+g_{l m} \Gamma_{i k}^{m}, \\
& g_{k l, i}=g_{m l} \Gamma_{k i}^{m}+g_{k m} \Gamma_{l i}^{m} .
\end{aligned}
$$

Since the technique is similar to the classical one, then we give formulas without justification:

$$
g_{i k, l}+g_{l i, k}-g_{k l, i}=g_{m k} S_{i l}^{m}+g_{m l} S_{i k}^{m}+g_{i m} \Gamma_{k l}^{m}+g_{m i} \Gamma_{l k}^{m},
$$

where $S_{i l}^{m}=\Gamma_{i l}^{m}-\Gamma_{l i}^{m}$ is torsion tensor, and we have

$$
\begin{aligned}
g_{i m} & \left(\Gamma_{k l}^{m}+\Gamma_{l k}^{m}\right) \\
& =g_{i k, l}+g_{l i, k}-g_{k l, i}+g_{k m} S_{l i}^{m}+g_{l m} S_{k i}^{m}, \\
\Gamma_{k l}^{p} & +\Gamma_{l k}^{p} \\
& =g^{p i}\left(g_{i k, l}+g_{l i, k}-g_{k l, i}+g_{k m} S_{l i}^{m}+g_{l m} S_{k i}^{m}\right),
\end{aligned}
$$

and complementing the obvious equation-definition $\Gamma_{k l}^{p}-$ $\Gamma_{l k}^{p}=S_{k l}^{p}$, then we obtain:

$$
\begin{aligned}
\Gamma_{k l}^{p}= & \frac{1}{2} g^{p i}\left(g_{i k, l}+g_{l i, k}-g_{k l, i}+g_{k m} S_{l i}^{m}+g_{l m} S_{k i}^{m}\right) \\
& +\frac{1}{2} S_{k l}^{p} .
\end{aligned}
$$

Then we introduce the notation and from the last formula we see that

$$
P_{k l}^{p}=\frac{1}{2} g^{p i}\left(g_{i k, l}+g_{l i, k}-g_{k l, i}\right)
$$

is geometric object and

$$
L_{k l}^{p} \equiv \frac{1}{2} S_{k l}^{p}+\frac{1}{2} g^{p i}\left(g_{k m} S_{l i}^{m}+g_{l m} S_{k i}^{m}\right)
$$

is tensor.

The geometric object $\Gamma_{k l}^{p}$, which generate connection space, is completely determined by the tensors $g_{i k}$ and $S_{i k}^{m}$. Therefore the connection $\Gamma_{k l}^{p}$ is the sum of a geometric object $P_{k l}^{p}$ which is composed of derivatives of the metric tensor $g_{i k}$ and tensor $L_{k l}^{p}$ is compiled of $g_{i k}$ and the tensor $S_{k l}^{m}$, namely,

$$
\Gamma_{k l}^{p}=P_{k l}^{p}+L_{k l}^{p}
$$

Remark 2. Tensor $L_{k l}^{p}$ represents the sum of two tensors: symmetric $(1 / 2) g^{p i}\left(g_{k m} S_{l i}^{m}+g_{l m} S_{k i}^{m}\right)$ and torsion $(1 / 2) S_{k l}^{p}$.

Remark 3. It is not difficult to prove the relation:

$$
\Gamma_{p l}^{p}=\frac{1}{2} g_{i p, l} g^{i p}=\frac{1}{\sqrt{g}} \frac{\partial \sqrt{g}}{\partial x^{l}}, \quad \text { where } g=\operatorname{det}\left|g_{i k}\right| \text {. }
$$

The next step in building a geometric theory is the consideration of the parallel transport tensor-vector $A^{i}$, which is given by

$$
d A^{i}=-\Gamma_{p l}^{i} A^{p} d x^{l}
$$

where the coefficients $\Gamma_{p l}^{i}$ are the connection of space.

As further arguments are similar to the classic and often repeated them, the presentation of intermediate results will wear schematic character.

By a covariant derivative of $u_{i}$ with respect to $l$ we mean

$$
\begin{aligned}
& u_{i ; l} \equiv u_{i, l}-\Gamma_{i l}^{k} u_{k}, \\
& u_{; l}^{i} \equiv u_{, l}^{i}+\Gamma_{k l}^{i} u^{k} .
\end{aligned}
$$

Then we consider the difference

$$
u_{i ; l}-u_{l ; i}=u_{i, l}-u_{l ; i}-S_{i l}^{k} u_{k} .
$$

During the transition along a parallelogram in the image to the original polygon the gap $Z^{k}$ is formed (breaking the circuit), which can be estimated as follows, up to the $2 \mathrm{nd}$ order of smallness relative to sides of a parallelogram:

$$
Z^{k}=S_{i j}^{k} A^{i} B^{j} \tau^{2}
$$

It is the result of coagulation at the torsion tensor with vector parties $A^{i} \tau$ and $B^{j} \tau$ that express geodetic displacement. The basic geometric meaning of the torsion tensor is an estimate of the gap (up to 2nd order) at which the open loop shrinks, if the torsion is zero, then the gap will not infinitesimal second but higher order smallness.

Next, we consider the difference of the second order derivatives:

$$
u_{i ; l ; k}-u_{i ; k ; l}=R_{k l i}^{p} u_{p}+S_{k l}^{q} u_{i ; q}
$$

where we identified

$$
R_{k l i}^{p} \equiv \Gamma_{i k, l}^{p}-\Gamma_{i l, k}^{p}+\Gamma_{q l}^{p} \Gamma_{i k}^{q}-\Gamma_{q k}^{p} \Gamma_{i l}^{q} .
$$

$R_{k l i}^{p}$ is curvature tensor.

Similarly, we have

$$
u_{; l ; k}^{i}-u_{; k ; l}^{i}=-R_{k l p}^{i} u^{p}+S_{k l}^{q} u_{; q}^{i} .
$$

Remark 4. Also possible and slightly different way of definition a covariant derivative, namely, the absolute differential $D A^{i}$, is first determined using the formula

$$
D A^{i} \equiv \sim d A^{i}+\Gamma_{j k}^{i} A^{j} d x^{k}=\left(A_{, k}^{i}+\Gamma_{j k}^{i} A^{j}\right) d x^{k} .
$$


Absolute differential can be defined as derivative coefficients, all the results obtained with this approach to the construction is identical with the analysis, which has been made above. Then more clearly we can assert that for any space to possess absolute parallelism it is a necessary and sufficient condition that curvature tensor be identity vanishing (recall that the space is called with absolute parallelism if the result of the parallel transport of an arbitrary tensor-vector does not depend on the choice of path for all points of space). The proof of this theorem is generally known, we note only that it follows from the formula:

$$
\widetilde{D} D A^{i}-D \widetilde{D} A^{i}=-R_{k l p}^{i} A^{p} \widetilde{d} x^{k} d x^{l}
$$

which we could get by folding (24) with $\widetilde{d} x^{k} d x^{l}$.

All the constructions outlined above are general in nature without specifying space; further we will investigate the structure of the tensor $R_{i k l}^{p}$. So, by definition of (23) we have

$$
R_{i k l}^{p}=\Gamma_{l i, k}^{p}-\Gamma_{l k, i}^{p}+\Gamma_{q k}^{p} \Gamma_{l i}^{q}-\Gamma_{q i}^{p} \Gamma_{l k}^{q} .
$$

Then we use (16) and obtain

$$
\begin{aligned}
R_{i k l}^{p}= & P_{l i, k}^{p}+L_{l i, k}^{p}-P_{l k, i}^{p}+L_{l k, i}^{p}+\left(P_{q k}^{p}+L_{q k}^{p}\right)\left(P_{l i}^{p}+L_{l i}^{q}\right) \\
& -\left(P_{q i}^{p}+L_{q i}^{p}\right)\left(P_{l k}^{p}+L_{l k}^{q}\right) \\
= & P_{l i, k}^{p}-P_{l k, i}^{p}+P_{q k}^{p} P_{l i}^{q}-P_{q i}^{p} P_{l k}^{q}+L_{l i, k}^{p}-L_{l k, i}^{p} \\
& +P_{q k}^{p} L_{l i}^{q}+P_{l i}^{q} L_{q k}^{p}-P_{q i}^{p} L_{l k}^{q}-P_{l k}^{q} L_{q i}^{p} \\
& +L_{q k}^{p} L_{l i}^{q}-L_{q i}^{p} L_{l k}^{q} .
\end{aligned}
$$

Next we introduce the notation:

$$
P_{i k l}^{p} \equiv P_{l i, k}^{p}-P_{l k, i}^{p}+P_{q k}^{p} P_{l i}^{q}+P_{q i}^{p} P_{l k}^{q}
$$

is a tensor like the Riemann curvature tensor, composed of the metric tensor and its derivatives. Consider the following:

$$
Z_{i k l}^{p} \equiv L_{q k}^{p} L_{l i}^{q}-L_{q i}^{p} L_{l k}^{q}
$$

is tensor and

$$
T_{i k l}^{p} \equiv L_{l i, k}^{p}-L_{l k, i}^{p}+P_{q k}^{p} L_{l i}^{q}+P_{l i}^{q} L_{q k}^{p}-P_{q i}^{p} L_{l k}^{q}-P_{l k}^{q} L_{q i}^{p}
$$

is tensor.

If we take into account that $R_{i k l}^{p}$ is tensor, the last assertion is obvious. It is interesting to obtain this important result in another way; namely,

$$
\begin{aligned}
T_{i k l}^{p}= & L_{l i ; k}^{p}-L_{l k ; i}^{p}-L_{l i}^{q} \Gamma_{q k}^{p}+L_{q i}^{p} \Gamma_{l k}^{q}+L_{l q}^{p} \Gamma_{i k}^{q}+L_{l k}^{q} \Gamma_{q i}^{p} \\
& -L_{q k}^{p} \Gamma_{l i}^{q}-L_{l q}^{p} \Gamma_{k i}^{q}+P_{q k}^{p} L_{l i}^{q}+P_{l i}^{q} L_{q k}^{q} \\
& -P_{q i}^{p} L_{l k}^{q}-P_{l k}^{q} L_{q i}^{p} \\
= & L_{l i ; k}^{p}-L_{l k ; i}^{p}-L_{l i}^{q} L_{q k}^{p}+L_{q i}^{p} L_{l k}^{q}+L_{l k}^{q} L_{q i}^{p} \\
& -L_{q k}^{p} L_{l i}^{q}+L_{l q}^{p} S_{i k}^{q}
\end{aligned}
$$

is obviously the tensor because the absolute derivatives have tensor character.

We introduce the notation

$$
M_{i k l}^{p} \equiv T_{i k l}^{p}+Z_{i k l}^{p}
$$

then we obtain

$$
M_{i k l}^{p}=L_{l i ; k}^{p}-L_{l k ; i}^{p}+L_{l q}^{p} S_{i k}^{q}+L_{q i}^{p} L_{l k}^{q}-L_{q k}^{p} L_{l i}^{q},
$$

and in the new notation

$$
R_{i k l}^{p}=P_{i k l}^{p}+M_{i k l}^{p} .
$$

Formula (35) shows that the curvature tensor, in general, cases can be represented as the sum of two tensors (such representation is not accidental, it is associated with a physical description of the field; roughly speaking, in the absence of gravitational fields tensor, $M_{i k l}^{p}$ is not equal to zero). Although the formula (34) gives a qualitative representation of the geometric structure, it is a little convenient, since it reenters the values of $\Gamma_{j k}^{i}$.

Further, we establish the equation, which is similar to equation of Ricci-Jacobi

$$
\begin{aligned}
R_{i k l}^{p}+R_{k l i}^{p}+R_{l i k}^{p}= & S_{i k, l}^{p}+S_{k l, i}^{p}+S_{l i, k}^{p}+\Gamma_{q k}^{p} S_{l i}^{q}+\Gamma_{q k}^{p} S_{i k}^{q}+\Gamma_{q i}^{p} S_{k l}^{q} \\
= & S_{i k ; l}^{p}+\Gamma_{i l}^{q} S_{q k}^{p}+\Gamma_{k l}^{q} S_{i q}^{p}+S_{k l ; i}^{p}+\Gamma_{k i}^{q} S_{q l}^{p}+\Gamma_{l i}^{q} S_{k q}^{p} \\
& +S_{l i ; k}^{p}+\Gamma_{l k}^{q} S_{q i}^{p}+\Gamma_{i k}^{q} S_{l q}^{p} \\
= & S_{i k ; l}^{p}+S_{k l ; i}^{p}+S_{l i ; k}^{p} \\
& +S_{l q}^{p} S_{i k}^{q}+S_{k q}^{p} S_{l i}^{q}+S_{i q}^{p} S_{k l}^{q} .
\end{aligned}
$$

It is easy to prove the equations

$$
\begin{gathered}
S_{j p}^{i} S_{k i}^{p}+S_{k p}^{i} S_{i j}^{p}=0, \\
S_{i p}^{i} S_{j k}^{p}=0
\end{gathered}
$$

and as a consequence, we obtain the equation

$$
S_{j p}^{i} S_{k i}^{p}+S_{k p}^{i} S_{i j}^{p}+S_{i p}^{i} S_{j k}^{p}=0 .
$$

\section{The Study of Geodesics in the Space with Torsion}

Formula (13) consists of three summand of various kinds.

The sum (14) is a geometric object second valence; its components are converted by a formula similar to the one that takes place for the connection:

$$
P_{k^{\prime} l^{\prime}}^{p^{\prime}}=P_{k l}^{p} \frac{\partial x^{p^{\prime}}}{\partial x^{p}} \frac{\partial x^{k}}{\partial x^{k^{\prime}}} \frac{\partial x^{l}}{\partial x^{l^{\prime}}}+\frac{\partial^{2} x^{p}}{\partial x^{k^{\prime}} \partial x^{l^{\prime}}} \frac{\partial x^{p^{\prime}}}{\partial x^{p}},
$$

but note that in this space, this "connection" does not satisfy the "torsion condition" $\left(S_{j k}^{i} \equiv \Gamma_{j k}^{i}-\Gamma_{k j}^{i}\right)$. 
Value $(1 / 2) S_{k l}^{p}$ : this tensor can be to be used as a "connection," but then this "connection" is not used to satisfy the compatibility condition.

Value

$$
M_{k l}^{p} \equiv \frac{1}{2} g^{p i}\left(g_{k m} S_{l i}^{m}+g_{l m} S_{k i}^{m}\right)
$$

is a tensor that expresses the combined effect of the metric and torsion.

First of all, it is easy to show that the element $(1 / 2) S_{k l}^{p}$ does not affect the geodesic, since by the asymmetry of the torsion it is not included in the equations of geodesic lines:

$$
\frac{d^{2} x^{k}}{d \tau^{2}}=-\Gamma_{i j}^{k} \frac{d x^{i}}{d \tau} \frac{d x^{j}}{d \tau}
$$

that is, (6) will be determined only by sum $P_{k l}^{p}+M_{k l}^{p}$. In (6) $\tau$ is the canonical parameter, that is the vector $d x^{i} / d \tau$ is parallel transported vector. For no isotropic geodesic the length of arc $s$ is a canonical parameter, for geodetic related to $s$ takes place in differential equations:

$$
\frac{d^{2} x^{k}}{d s^{2}}=-\Gamma_{i j}^{k} \frac{d x^{i}}{d s} \frac{d x^{j}}{d s} .
$$

Definition 5. The line is called a geodesic if any tangent to this line at some point vector remains tangent to it at the parallel transport along it.

Theorem 6. The equations of geodesic lines in space $Y^{n}$ is determined by the geometric object in form of the sum:

$$
\begin{aligned}
P_{k l}^{p}+M_{k l}^{p}= & \frac{1}{2} g^{p i}\left(g_{i k, l}+g_{l i, k}-g_{k l, i}\right) \\
& +\frac{1}{2} g^{p i}\left(g_{k m} S_{l i}^{m}+g_{l m} S_{k i}^{m}\right) .
\end{aligned}
$$

In case classical Riemannian space geodetic lines have known extreme properties, in this case analogical properties of geodesic require additional research.

Thus, for a no isotropic geodesic with canonical parameter $\operatorname{arclength} s$, we have differential equations:

$$
\frac{d^{2} x^{k}}{d s^{2}}=-\Gamma_{i j}^{k} \frac{d x^{i}}{d s} \frac{d x^{j}}{d s} .
$$

Consider the problem of calculating the variation of the arc length.

Let a nonisotropic curve $x^{i}=x^{i}(t), t \in\left[t_{1} ; t_{2}\right]$. We calculate the variation of length $\delta S$ of the curve $S$ :

$$
\begin{aligned}
\delta S & =\int_{t_{1}}^{t_{2}} \delta \sqrt{g_{i j} \frac{d x^{i}}{d t} \frac{d x^{j}}{d t}} d t \\
& =\int_{t_{1}}^{t_{2}} \frac{\delta\left(g_{i j}\left(d x^{i} / d t\right)\left(d x^{j} / d t\right)\right)}{2 \sqrt{g_{i j}\left(d x^{i} / d t\right)\left(d x^{j} / d t\right)}} d t \\
\delta\left(g_{i j} \frac{d x^{i}}{d t} \frac{d x^{j}}{d t}\right) & =g_{i j} \widetilde{D} \frac{d x^{i}}{d t} \frac{d x^{j}}{d t}+g_{i j} \frac{d x^{i}}{d t} \widetilde{D} \frac{d x^{j}}{d t} \\
& =2 g_{i j} \frac{d x^{i}}{d t} \widetilde{D} \frac{d x^{j}}{d t},
\end{aligned}
$$

$$
\begin{aligned}
& \widetilde{D} \frac{d x^{j}}{d t}=\delta \frac{d x^{j}}{d t}+\Gamma_{p k}^{j} \frac{d x^{p}}{d t} \delta x^{k}, \\
& D \frac{\delta x^{j}}{d t}=\frac{d}{d t} \delta x^{j}+\Gamma_{k p}^{j} \frac{d x^{k}}{d t} \delta x^{p}, \\
& \widetilde{D} \frac{d x^{j}}{d t}=D \frac{\delta x^{j}}{d t}+S_{p k}^{j} \frac{d x^{p}}{d t} \delta x^{k},
\end{aligned}
$$

where $\widetilde{D}$ denotes the absolute differential through the parameter curves of the family at a constant value $t$ and $D$ is absolute differential charge small displacement $d t$ curve at a constant parameter of the family; then

$$
\begin{aligned}
\delta\left(g_{i j} \frac{d x^{i}}{d t} \frac{d x^{j}}{d t}\right)= & 2 g_{i j} \frac{d x^{i}}{d t}\left(D \frac{\delta x^{j}}{d t}+S_{p k}^{j} \frac{d x^{p}}{d t} \delta x^{k}\right), \\
\delta s= & \int_{t_{1}}^{t_{2}} g_{i j} \frac{d x^{i}}{d s} D \delta x^{j} \\
& +\int_{t_{1}}^{t_{2}} g_{i j} S_{p k}^{j} \frac{d x^{i}}{d s} d x^{p} \delta x^{k} \\
= & \int_{t_{1}}^{t_{2}} D\left(g_{i j} \frac{d x^{i}}{d s} \delta x^{j}\right) \\
& -\int_{t_{1}}^{t_{2}} g_{i j} D \frac{d x^{i}}{d s} \delta x^{j} \\
& +\int_{t_{1}}^{t_{2}} g_{i j} S_{p k}^{j} \frac{d x^{i}}{d s} d x^{p} \delta x^{k}
\end{aligned}
$$

if the ends of the variable curve are fixed, then

$$
\delta s=\int_{t_{1}}^{t_{2}}\left(g_{i j} S_{p k}^{j} \frac{d x^{i}}{d t} d x^{p} \delta x^{k}-g_{i j} D \frac{d x^{i}}{d t} \delta x^{j}\right) ;
$$

if the considered curve has fixed length (analytically $\delta s=0$ ), then we obtain

$$
\int_{t_{1}}^{t_{2}}\left(g_{i j} S_{p k}^{j} \frac{d x^{i}}{d s} d x^{p} \delta x^{k}-g_{i j} D \frac{d x^{i}}{d s} \delta x^{j}\right)=0 .
$$

Using the fundamental lemma calculus of variations, it follows that

$$
g_{i k} S_{p j}^{k} \frac{d x^{i}}{d s} d x^{p}-g_{i j} D \frac{d x^{i}}{d s}=0 .
$$

This equation means that the tangent vector $\xi^{q}$ of the curve is transported according to the law $D \xi^{q} g^{j q} g_{i k} S_{p j}^{k} \xi^{i} d x^{p}$, which means that $s$ is not geodesic curve. Conversely, the variation of the length of the geodesic lines is

$$
\delta s=\int_{t_{1}}^{t_{2}} g_{i j} S_{p k}^{j} \frac{d x^{i}}{d t} d x^{p} \delta x^{k} .
$$

Properties in the new geodesic geometry defined by means of two tensors $g_{i k}$ and $S_{i k}^{j}$ differ greatly from similar properties in Riemann geometry. The theorem is proved. 
Theorem 7. In order a line in space $Y^{n}$ (that is generated by $g_{i k}$ and $S_{i k}^{j}$ ) was geodetic it is necessary and sufficient that a variation of the arc this line was equaled:

$$
\int_{t_{1}}^{t_{2}} g_{i j} j_{p k}^{j} \frac{d x^{i}}{d t} d x^{p} \delta x^{k}
$$

Consequence. In the case of spaces with affine connection is known that there is a gap (breach) of closure during the transition from the original to the image and vice versa, in the case of an infinitely small contour (determined up to the second order relative to $\tau$ ). If you specify the torsion tensor $S_{i j}^{k}$ at the corresponding point, then if this gap is denoted by $\Psi^{k}$, then $\Psi^{k}=S_{i j}^{k} A^{i} B^{j} \tau^{2}$, where the parallelogram $A^{i} \tau$ and $B^{j} \tau$ shrink to a point at $\tau \rightarrow 0$. In this case, we have the formula for square of the length: $|\Psi|^{2}=g_{p q} S_{i j}^{p} A^{i} B^{j} S_{k l}^{q} A^{k} B^{l} \tau^{4}$.

Theorem 8. Let classical Riemannian space be given (Riemannian manifold with Riemannian metric tensor $g_{i k}$ ) with the connection $P_{i j}^{k}$, connection Riemannian space. Let $Y^{n}$ be the space generated jointly and agreed on by metric $g_{i k}$ and torsion $S_{i j}^{k}$ tensors together with connection $\Gamma_{i j}^{k}$. To coincide the geodesics in classical Riemannian space with the geodesics in space $Y^{n}$ it is necessary and sufficient that the connections $P_{i j}^{k}$ and $\Gamma_{i j}^{k}$ be differed by tensor:

$$
\frac{1}{2} \frac{1}{n+1}\left(\delta_{i}^{k} S_{j l}^{l}+\delta_{j}^{k} S_{i l}^{l}\right)
$$

that is,

$$
\Gamma_{i j}^{k}-P_{i j}^{k}=\frac{1}{2} \frac{1}{n+1}\left(\delta_{i}^{k} S_{j l}^{l}+\delta_{j}^{k} S_{i l}^{l}\right) .
$$

Remark 9. The last summand in

$$
\begin{aligned}
\Gamma_{i j}^{k}= & \frac{1}{2} g^{k p}\left(g_{i p j l}+g_{j p, i}-g_{i j, p}+g_{i m} S_{j p}^{m}+g_{j m} S_{i p}^{m}\right) \\
& +\frac{1}{2} S_{i j}^{k},
\end{aligned}
$$

that is, $(1 / 2) S_{i j}^{k}$, is irrelevant (it is clear).

Proof. The necessity means that if the geodesics in classical Riemannian space coincide with the geodesics in space $Y^{n}$, then

$$
\Gamma_{i j}^{k}-P_{i j}^{k}=\frac{1}{2} \frac{1}{n+1}\left(\delta_{i}^{k} S_{j l}^{l}+\delta_{j}^{k} S_{i l}^{l}\right)
$$

Let the vector $A^{i}$ be tangent to any geodesic and $A^{i}$ is parallel transported to the connection $\Gamma_{k l}^{p}$ :

$$
\begin{aligned}
\Gamma_{k l}^{p}= & \frac{1}{2} g^{p i}\left(g_{i k, l}+g_{l i, k}-g_{k l, i}+g_{k m} S_{l i}^{m}+g_{l m} S_{k i}^{m}\right) \\
& +\frac{1}{2} S_{k l}^{p},
\end{aligned}
$$

and a vector $B^{i}$ is parallel transported to the connection $P_{k l}^{p}$ :

$$
P_{k l}^{p}=\frac{1}{2} g^{p i}\left(g_{i k, l}+g_{l i, k}-g_{k l, i}\right) .
$$

It was found that the first geodesic connections coincide with geodesic connection $P_{k l}^{p}+M_{k l}^{p}$; here $M_{i j}^{k}$ is an arbitrary symmetric tensor. Since both vectors are tangential, then

$$
B^{i}=a A^{i}
$$

where the coefficient $a$ is variable and $a \neq 0$. Tensor-vector $A^{i}$ is given by

$$
\begin{aligned}
& d A^{k}=-\left(P_{i j}^{k}+M_{i j}^{k}\right) A^{i} d x^{j}, \\
& d B^{k}=-P_{i j}^{k} B^{i} d x^{j} .
\end{aligned}
$$

Then, we have

$$
\begin{aligned}
A^{k} d a+a d A^{k} & =-P_{i j}^{k} a A^{i} d x^{j}, \\
\frac{A^{k} d a}{a} & =M_{i j}^{k} A^{i} d x^{j} .
\end{aligned}
$$

Tangent vector $A^{i}$ can be written: $A^{i}=d x^{i} / d \tau$, where $\tau$ is the canonical parameter relative to the connection $P_{k l}^{p}+M_{k l}^{p}$.

Then, after dividing by $d \tau$, we have

$$
\frac{d \ln a}{d \tau} A^{k}=M_{i j}^{k} A^{i} A^{j} .
$$

Since geodesic lines can be carried out through any point and in any direction, then equality must be true at any point and for any vector $A^{i}$, the functional dependence of the point and direction, obviously there. and $l$ :

The last equality is multiplied by $A^{l}$ and alternated by $k$

$$
A^{l} M_{i j}^{k} A^{i} A^{j}-A^{k} M_{i j}^{l} A^{i} A^{j}=0,
$$

or

$$
\delta_{m}^{l} M_{i j}^{k} A^{i} A^{j} A^{m}-\delta_{m}^{k} M_{i j}^{l} A^{i} A^{j} A^{m}=0,
$$

where we denote $\delta_{m}^{l}=g_{m p} g^{p l}$. This equation must hold identically with respect to vectors $A^{1}, \ldots, A^{n}$, consequently, after summed similar summands all the coefficients of the cubic form must vanish. We compute the total coefficient:

$$
\begin{aligned}
& \delta_{m}^{l} M_{i j}^{k}-\delta_{m}^{k} M_{i j}^{l}+\delta_{i}^{l} M_{j m}^{k}-\delta_{i}^{k} M_{j m}^{l}+\delta_{j}^{l} M_{m i}^{k}-\delta_{j}^{k} M_{m i}^{l} \\
& =0 .
\end{aligned}
$$

Then we contracted tensor by indices $l$ and $j$. Since $\delta_{i}^{i}=n$, we have

$$
M_{i j}^{k}=\frac{1}{n+1}\left(\delta_{i}^{k} M_{j l}^{l}+\delta_{j}^{k} M_{i l}^{l}\right) .
$$


All calculations above are correct for any symmetrical tensor $M_{i j}^{k}$.

All calculations presented above do not take into account the specificity of the tensor $M_{i j}^{k}$; then, let $M_{k l}^{p} \equiv$ $(1 / 2) g^{p i}\left(g_{k m} S_{l i}^{m}+g_{l m} S_{k i}^{m}\right)$; then $M_{k l}^{l} \equiv(1 / 2) S_{k l}^{l}$.

We obtained

$$
\Gamma_{i j}^{k}-P_{i j}^{k}=\frac{1}{2} \frac{1}{n+1}\left(\delta_{i}^{k} S_{j l}^{l}+\delta_{j}^{k} S_{i l}^{l}\right) .
$$

The necessity is proof.

Remark 10. In the beginning we considered more general situation and then specified tensor $M_{i j}^{k}$.

We prove the sufficiency. We assume that $\Gamma_{i j}^{k}-P_{i j}^{k}=$ $(1 / 2)(1 /(n+1))\left(\delta_{i}^{k} S_{j l}^{l}+\delta_{j}^{k} S_{i l}^{l}\right)$; we have to show that the geodesics coincide.

We again use (61); we have $A^{k} d a / a=M_{i j}^{k} A^{i} d x^{j}$.

Tangent vector $A^{i}$ can be written: $A^{i}=d x^{i} / d \tau$, where $\tau$ is the canonical parameter relative to the connection $\Gamma_{i j}^{k}$.

Since along the curve $S_{i l}^{l} A^{i}$ there is a definite function of the parameter $\tau$, then it will find $\ln a$ after integration with up to a constant $a$, but only up to a constant factor (we can integrate). Therefore, the vector is found to be $B^{i}=a A^{i}$ and all geodesics coincide. The sufficiency is proved. The theorem is proved.

\section{The Theory of Hypersurfaces}

First we formulate a well-known classical result on the derivation formulas of hypersurface in Riemannian torsionfree space, where the connection is uniquely generated by the metric.

Formal statement of Gauss-Codazzi equations in space with symmetrical connection, $S_{i j}^{k}=0$. Assume that $i: M \subset P$ is $n$-dimensional embedded submanifold of a Riemannian manifold $P$ of dimension $n+p$. There is a natural inclusion of the tangent bundle of $M$ into that of $P$ by the pushforward, and the cokernel is the normal bundle of $M: 0 \rightarrow T_{x} \rightarrow$ $\left.T_{x} P\right|_{M} \rightarrow T_{x}^{\perp} M \rightarrow 0$. The metric splits this short exact sequence, and so $\left.T P\right|_{M}=T M \oplus T^{\perp} M$.

The Levi-Civita connection $\nabla^{\prime}$ of $P$ decomposes into tangential and normal components. For each $X \in T M$ and vector field $Y$ on $M, \nabla_{X}^{\prime} Y=T\left(\nabla_{X}^{\prime} Y\right)+\perp\left(\nabla_{X}^{\prime} Y\right)$. Let it be $\nabla_{X} Y=T\left(\nabla_{X}^{\prime} Y\right), \alpha(X, Y)=\perp\left(\nabla_{X} Y\right)$. Gauss' formula asserts that $\nabla_{X}$ is Levi-Civita connection on $M$, and $\alpha$ is a symmetric form with values into the normal bundle. It is also referred to as the second fundamental form. An immediate corollary is the Gauss equation. For $X, Y, Z, W \in T M$,

$$
\begin{aligned}
\left\langle R^{\prime}(X, Y) Z, W\right\rangle= & \langle R(X, Y) Z, W\rangle \\
& +\langle\alpha(X, Z), \alpha(Y, W)\rangle \\
& -\langle\alpha(Y, Z), \alpha(X, W)\rangle,
\end{aligned}
$$

where $R^{\prime}$ is the Riemann curvature tensor of $P$ and $R$ is that of $M$.
There are thus a pair of connections: $\nabla$, defined on the tangent bundle of $M$, and $D$, defined on the normal bundle of $M$. These combine to form a connection on any tensor product of copies of $T M$ and $T \perp M$. In particular, they defined the covariant derivative of $\alpha$ :

$$
\begin{aligned}
\left(\nabla_{X}^{\prime \prime} \alpha\right)(Y, Z)= & D_{X}(\alpha(Y, Z))-\alpha\left(\nabla_{X} Y, Z\right) \\
& -\alpha\left(Y, \nabla_{X} Z\right) .
\end{aligned}
$$

Codazzi equation is

$$
\perp\left\langle R^{\prime}(X, Y) Z\right\rangle=\left(\nabla_{X}^{\prime \prime} \alpha\right)(Y, Z)-\left(\nabla_{Y}^{\prime \prime} \alpha\right)(X, Z) .
$$

The above formulas also hold for immersions, because every immersion is, in particular, a local embedding. The important assumption in the theory stated above is that connection is symmetrical, so analog of second form of hypersurface is symmetrical and we could obtain the derivation formulas of hypersurface.

In our case we do not assume any conditions about the connection symmetry, so below we study much more complicated problem.

A similar manner as in the Riemannian space can be developed as a theory of hypersurfaces, so in the metric space with torsion a theory of hypersurfaces can be constructed. But, due to the presence of torsion, in these cases there is a significant difference. For example, the derivation equations (analog Peterson Codazzi equations) take a more complicated form, in which there are new summands, which are caused by the presence of torsion in the space.

We make a study of the hypersurfaces $Y^{n-1}$ in a metric space with torsion $Y^{n}$. We are assuming that the hypersurface is defined by a system of equations:

$$
x^{i}=x^{i}\left(y^{1}, \ldots, y^{n-1}\right)
$$

and the rank of the matrix $\left[\partial x^{i} / \partial y^{\alpha}\right]$ equals $n-1$. The metric tensor of hypersurface $Y^{n-1}$ is given by

$$
a_{\alpha \beta}=g_{i j} \frac{\partial x^{i}}{\partial y^{\alpha}} \frac{\partial x^{j}}{\partial y^{\beta}}
$$

Then we obtain the formula for tensor of torsion $T_{\alpha \beta}^{\gamma}$ of hypersurface $Y^{n-1}$ (assuming that functions $x^{i}\left(y^{1}, \ldots, y^{n-1}\right.$ ) are smooth enough). Let $G_{\beta \gamma}^{\alpha}$ be the connection of $Y^{n-1}$ and we assume that $G_{\beta \gamma}^{\alpha}$ express via metric $a_{\alpha \beta}$ and torsion $T_{\alpha \beta}^{\gamma}$ similarly to as the connection $\Gamma_{i j}^{k}$ express by means of $g_{i j}$ and $S_{i j}^{k}$, we have:

$$
\begin{aligned}
& G_{\beta \gamma}^{\alpha} \\
& =\frac{1}{2}\left(a^{\alpha \eta}\left(a_{\beta \eta, \gamma}+a_{\gamma \eta, \beta}+a_{\beta \gamma, \eta}+a_{\beta \mu} T_{\gamma \eta}^{\mu}+a_{\gamma \mu} T_{\beta \eta}^{\mu}\right)+T_{\gamma \beta}^{\alpha}\right),
\end{aligned}
$$


$G_{\beta \gamma}^{\alpha}$ is geometric object and is subjected to the law of the transformation from one coordinate system $u^{\alpha}$ to another $u^{\alpha^{\prime}}$ by the formula:

$$
G_{\beta \gamma}^{\alpha}=G_{\beta^{\prime} \gamma^{\prime}}^{\alpha^{\prime}} \frac{\partial u^{\alpha}}{\partial u^{\alpha^{\prime}}} \frac{\partial u^{\beta^{\prime}}}{\partial u^{\beta}} \frac{\partial u^{\gamma^{\prime}}}{\partial u^{\gamma}}+\frac{\partial u^{\alpha}}{\partial u^{\alpha^{\prime}}} \frac{\partial^{2} u^{\alpha^{\prime}}}{\partial u^{\beta} \partial u^{\gamma}} .
$$

Then we assume that connection $G_{\beta \gamma}^{\alpha}$ of $Y^{n-1}$ are associated with connection $\Gamma_{i j}^{k}$ of $Y^{n}$ mean of formula:

$$
G_{\beta \gamma}^{\alpha} \frac{\partial x^{k}}{\partial u^{\alpha}}=\Gamma_{i j}^{k} \frac{\partial x^{i}}{\partial u^{\beta}} \frac{\partial x^{j}}{\partial u^{\gamma}}+\frac{\partial^{2} x^{k}}{\partial u^{\beta} \partial u^{\gamma}} .
$$

We obtain

$$
\begin{aligned}
\frac{1}{2}\left(a^{\alpha \eta}\left(a_{\beta \eta, \gamma}+a_{\gamma \eta, \beta}+a_{\beta \gamma, \eta}+a_{\beta \mu} T_{\gamma \eta}^{\mu}+a_{\gamma \mu} T_{\beta \eta}^{\mu}\right)+T_{\gamma \beta}^{\alpha}\right) \frac{\partial x^{k}}{\partial u^{\alpha}} \\
=\frac{1}{2}\left(g^{k n}\left(g_{n i, j}+g_{n j, i}-g_{i j, n}+g_{i m} S_{j n}^{m}+g_{j m} S_{i n}^{m}\right)+S_{i j}^{k}\right) \\
\quad \times \frac{\partial x^{i}}{\partial u^{\beta}} \frac{\partial x^{j}}{\partial u^{\gamma}}+\frac{\partial^{2} x^{k}}{\partial u^{\beta} \partial u^{\gamma}} .
\end{aligned}
$$

By permuting indices, we have the next formula for the torsion tensor of hypersurface $Y^{n-1}$ :

$$
T_{\alpha \beta}^{\gamma}=a^{\gamma \eta} g_{p q} S_{i j}^{p} \frac{\partial x^{i}}{\partial y^{\alpha}} \frac{\partial x^{j}}{\partial y^{\beta}} \frac{\partial x^{q}}{\partial y^{\eta}}
$$

using tensors $a_{\alpha \beta}$ and $T_{\alpha \beta}^{\gamma}$, both metric and torsion, we can explore the geometry of the space hypersurface $Y^{n-1}$.

The connection of $Y^{n-1}$ will be determined by the formula (73).

Below we use the mixed tensors values which enumerated two types of indices, while Latin indices refer to the containing space $Y^{n}$ and are responsive to the coordinate transformation $x^{i}$, and Greek indices belong to the space hypersurface $Y^{n-1}$ and are responsive to the coordinate transformation $y^{\alpha}$.

The index $i$ is not responsive to the coordinate $y^{\alpha}$ transformation into $Y^{n-1}$, and the index $\alpha$ does not respond to the coordinate $x^{i}$ transformation in $Y^{n}$. For example, the formula to calculate the covariant derivative of a mixed tensor:

$$
\begin{aligned}
A_{j \beta ; \gamma}^{i \alpha}= & A_{j \beta, \gamma}^{i \alpha}+\Gamma_{p k}^{i} A_{j \beta}^{p \alpha} \frac{\partial x^{k}}{\partial y^{\gamma}}-\Gamma_{j q}^{p} A_{p \beta}^{i \alpha} \frac{\partial x^{q}}{\partial y^{\gamma}}+G_{\eta \gamma}^{\alpha} A_{j \beta}^{i \eta} \\
& -G_{\beta \gamma}^{\eta} A_{j \eta}^{i \alpha} .
\end{aligned}
$$

The direct calculations lead us to formulas

$$
\begin{aligned}
& u_{i ; \alpha ; \beta}-u_{i ; \beta ; \alpha}=R_{k l i}^{p} u_{p} \frac{\partial x^{l}}{\partial y^{\alpha}} \frac{\partial x^{k}}{\partial y^{\beta}}+S_{k l}^{q} u_{i ; q} \frac{\partial x^{l}}{\partial y^{\alpha}} \frac{\partial x^{k}}{\partial y^{\beta}}, \\
& u_{\gamma ; \alpha ; \beta}-u_{\gamma ; \beta ; \alpha}=R_{\beta \alpha \gamma}^{\eta} u_{\eta}+T_{\beta \alpha}^{\eta} u_{\gamma ; \eta},
\end{aligned}
$$

where $R_{\beta \alpha \gamma}^{\eta}$ is curvature tensor of space $Y^{n-1}$ compiled by using the components of connection $G_{\beta \gamma}^{\alpha}$.

A further aim of our study is to obtain some analogs of Peterson-Kodachi equations. To do this, consider the system of values:

$$
\xi_{\alpha}^{i}=\frac{\partial x^{i}}{\partial y^{\alpha}}
$$

At each point of the hypersurface $Y^{n-1}$ we can build rapper consisting of the vectors:

$$
\xi_{1}^{i}, \ldots, \xi_{n-1}^{i}, \nu^{i}
$$

where $\xi_{1}^{i}, \ldots, \xi_{n-1}^{i}$ are linearly independent tangent vectors and $v^{i}$ is normal vector, defined since the metric and connectivity agreed.

Next we act formally; the idea is the same as in the classical case, and we will indicate significant new moments. We compute the derivative of the mixed tensors $\xi_{\alpha}^{i}$ :

$$
\xi_{\alpha ; \gamma}^{i}=\xi_{\alpha, \gamma}^{i}+\Gamma_{p q}^{i} \xi_{\alpha}^{p} \frac{\partial x^{q}}{\partial y^{\gamma}}-G_{\alpha \gamma}^{\eta} \xi_{\eta}^{i}
$$

In contrast to the case of torsion-free connection, we have the equality:

$$
\xi_{\alpha ; \gamma}^{i}-\xi_{\gamma ; \alpha}^{i}=S_{p q}^{i} \xi_{\alpha}^{p} \xi_{\gamma}^{q}+T_{\gamma \alpha}^{\eta} \xi_{\eta}^{i}
$$

Next, we permute the indices in equation

$$
0=a_{\alpha \beta ; \gamma}=\left(g_{i j} \xi_{\alpha}^{i} \xi_{\beta}^{j}\right)_{; \gamma}=g_{i j} \xi_{\alpha ; \gamma}^{i} \xi_{\beta}^{j}+g_{i j} \xi_{\alpha}^{i} \xi_{\beta ; \gamma}^{j}
$$

we obtain

$$
g_{i j} \xi_{\alpha ; \gamma}^{i} \xi_{\beta}^{j}=0
$$

Hence, we can write a decomposition:

$$
\xi_{\beta ; \alpha}^{i}=\pi_{\alpha \beta} \nu^{i}
$$

Remark 11. $\pi_{\alpha \beta}$ is tensor, which is similar to the second fundamental tensor of hypersurfaces $Y^{n-1}$, but its structure in this space is substantially different from the case of Riemannian spaces with zero torsion.

Then, we have obtained (by differentiating $g_{i j} \nu^{i} \xi_{\alpha}^{j}=0$ by $\gamma$ ) the formula:

$$
g_{i j} \nu_{; \gamma}^{i} \xi_{\alpha}^{i}=-\pi_{\gamma \alpha}
$$

Similarly, by differentiating $g_{i j} \nu^{i} \nu^{j}=1$ by $\gamma$, we obtain

$$
\nu_{; \gamma}^{i}=-a^{\eta \mu} \pi_{\mu \gamma} \xi_{\eta}^{i}
$$

Formulae (86) and (88) characterize the change of vectors in the small accompanying frame relative to this frame itself. 
Further, we obtain

$$
\begin{aligned}
\xi_{\beta ; \chi ; \lambda}^{i}-\xi_{\beta ; \lambda ; \chi}^{i}= & -R_{k l p}^{i} \xi_{\lambda}^{k} \xi_{\chi}^{l} \xi_{\beta}^{p}+R_{\lambda \chi \beta}^{\sigma} \xi_{\sigma}^{i}+T_{\lambda \chi}^{\sigma} \xi_{\beta ; \sigma}^{i} \\
= & \left(\pi_{\chi \beta ; \lambda}-\pi_{\lambda \beta ; \chi}\right) \nu^{i} \\
& -\left(\pi_{\chi \beta} \pi_{\eta \lambda} a^{\eta \sigma}-\pi_{\lambda \beta} \pi_{\eta \chi} a^{\eta \sigma}\right) \xi_{\sigma}^{i} .
\end{aligned}
$$

Equation (89) is multiplied by $g_{i j} \xi_{\alpha}^{j}$; we have

$$
R_{\alpha \lambda \chi \beta}=R_{i k l p} \xi_{\lambda}^{k} \xi_{\chi}^{l} \xi_{\beta}^{p} \xi_{\alpha}^{i}-\left(\pi_{\chi \beta} \pi_{\alpha \lambda}-\pi_{\lambda \beta} \pi_{\alpha \chi}\right) .
$$

Similarly, we derive a formula

$$
\begin{aligned}
v_{; \chi ; \lambda}^{i}-v_{; \lambda ; \chi}^{i} & =-R_{k l p}^{i} \xi_{\lambda}^{k} \xi_{\chi}^{l} \nu^{p}+T_{\lambda \chi}^{\sigma} \nu_{; \sigma}^{i} \\
& =\left(\pi_{\eta \lambda ; \chi} a^{\eta \sigma}-\pi_{\eta \chi ; \lambda} a^{\eta \sigma}\right) \xi_{\sigma}^{i} .
\end{aligned}
$$

We contract (89) with $g_{i j} \nu^{j}$; then

$$
-R_{i k l p} \xi_{\lambda}^{k} \xi_{\chi}^{l} \xi_{\beta}^{p} \nu^{i}+T_{\lambda \chi}^{\sigma} \pi_{\sigma \beta}=\pi_{\chi \beta ; \lambda}-\pi_{\lambda \beta ; \chi} .
$$

Formula (91) is multiplied by $g_{i j} \xi_{\alpha}^{j}$; we concluded that

$$
-R_{i k l p} \xi_{\lambda}^{k} \xi_{\chi}^{l} \nu^{p} \xi_{\alpha}^{i}+T_{\lambda \chi}^{\sigma} \pi_{\alpha \sigma}=\pi_{\alpha \lambda ; \chi}-\pi_{\alpha \chi ; \lambda} .
$$

Remark 12. If (91) contract with $g_{i j} \nu^{j}$, then we obtain identically zero equals zero.

Thus, we have the two types of formulas. Formula (90) does not contain the torsion tensor explicitly, but it is counted in the tensor $\pi_{\alpha \beta}$. In the formula (91) the torsion tensor of the hypersurface is present explicitly and in the form of coefficients of $\pi_{\alpha \beta}$ and appears in the calculation of the covariant derivative.

\section{Establish Some Important Relationships}

We consider the equation

$$
\begin{aligned}
S_{j k ; p ; q}^{i}-S_{j k ; q ; p}^{i}= & R_{q p j}^{t} S_{t k}^{i}+R_{q p k}^{t} S_{j t}^{i}-R_{q p t}^{i} S_{j k}^{t} \\
& +S_{q p}^{t} S_{j k ; t}^{i},
\end{aligned}
$$

or

$$
\begin{aligned}
& S_{j k ; p ; q}^{i}-S_{j k ; q ; p}^{i}-S_{q p}^{t} S_{j k ; t}^{i} \\
& \quad=R_{q p j}^{t} S_{t k}^{i}+R_{q p k}^{t} S_{j t}^{i}-R_{q p t}^{i} S_{j k}^{t} ;
\end{aligned}
$$

we contract these tensors by indices $i, q$; then the left side of this equation can be transformed into

$$
\begin{aligned}
& S_{j k ; p ; i}^{i}-S_{j k ; i p p}^{i}-S_{i p}^{t} S_{j k ; t}^{i} \\
& \quad=\left(S_{j k ; p}^{i}-S_{q p}^{i} S_{j k}^{q}\right)_{; i}-S_{j k ; i p}^{i}-S_{p q ; i}^{i} S_{j k}^{q} ;
\end{aligned}
$$

then, we contract this equation by indices $k, p$ and raising the index $j$; we obtained

$$
\begin{aligned}
\left(g^{k p}\right. & \left.g^{j s} S_{s k ; p}^{i}-g^{k p} g^{j s} S_{q p}^{i} S_{s k}^{q}\right)_{; i} \\
& -g^{k p} g^{j s} S_{s k ; i p}^{i}-g^{k p} g^{j s} S_{p q ; i}^{i} S_{s k}^{q} \\
= & g^{k p} g^{j s} R_{i p s}^{t} S_{t k}^{i}+g^{k p} g^{j s} R_{i p k}^{t} S_{s t}^{i} \\
& -g^{k p} g^{j s} R_{i p t}^{i} S_{s k}^{t} ;
\end{aligned}
$$

we introduce the notation

$$
\begin{aligned}
& H^{j i}=g^{k p} g^{j s} S_{s k ; p}^{i}-g^{k p} g^{j s} S_{q p}^{i} S_{s k}^{q}, \\
& F^{j p}=g^{k p} g^{j s} S_{s k ; i}^{i} .
\end{aligned}
$$

Then, without any loss of generality, we obtain the relations:

$$
\begin{aligned}
H_{; i}^{j i} & -F_{; i}^{j i}-g^{k p} g^{j s} S_{s k}^{q} F_{p q} \\
& =g^{k p} g^{j s} R_{i p s}^{t} S_{t k}^{i}+g^{k p} g^{j s} R_{i p k}^{t} S_{s t}^{i}-g^{k p} g^{j s} R_{i p t}^{i} S_{s k}^{t},
\end{aligned}
$$

where $F_{p q}=S_{p q ; i}^{i}$.

Suppose now that the identity Ricci-Jacobi run in a standard form, $R_{i k l}^{p}+R_{k l i}^{p}+R_{l i k}^{p}=0$; hence

$$
S_{i k ; l}^{p}+S_{k l ; i}^{p}+S_{l i ; k}^{p}+S_{l q}^{p} S_{i k}^{q}+S_{k q}^{p} S_{l i}^{q}+S_{i q}^{p} S_{k l}^{q}=0 .
$$

We contract this equation by indices $p, l$ and we found identity:

$$
S_{i k ; p}^{p}+S_{k p ; i}^{p}+S_{p i ; k}^{p}=0 .
$$

Next, we are assuming that $S_{i p}^{p}=\varphi_{i}$ and taking into account the identity $S_{i j}^{p} S_{p q}^{q}=0$, we obtain the following expression:

$$
S_{i j ; p}^{p}=\varphi_{i, j}-\varphi_{j, i} .
$$

Next if we put $S_{i j ; p}^{p}=0$, then it follows that $\varphi_{i, j}-\varphi_{j, i}=$ 0 and hence the value $S_{i p}^{p}$ can be expressed in terms of the partial derivative of the scalar $S_{i p}^{p}=\varphi_{i}=(\ln \psi)_{, i}$. System (99) takes the form

$$
\begin{aligned}
H_{; i}^{j i} & =g^{k p} g^{j s} R_{i p s}^{t} S_{t k}^{i}+g^{k p} g^{j s} R_{i p k}^{t} S_{s t}^{i}-g^{k p} g^{j s} R_{i p t}^{i} S_{s k}^{t}, \\
F^{i j} & =0 .
\end{aligned}
$$

We consider the tensor

$$
C^{i j k}=g^{p j} g^{q k} S_{p q}^{i}+g^{p k} g^{q i} S_{p q}^{j}+g^{p i} g^{q j} S_{p q}^{k}
$$

obvious that tensor is antisymmetric.

We have the equality

$$
\begin{aligned}
H^{j k}-H^{k j}= & C_{; i}^{i k j}+F^{j k}+g^{k p} g^{q s} S_{p q}^{t} S_{t s}^{j} \\
& -g^{j p} g^{q s} S_{p q}^{t} S_{t s}^{k} .
\end{aligned}
$$


By direct calculations we can conclude that

$$
\begin{aligned}
g^{k p} & g^{q s} S_{p q}^{t} S_{t s}^{j}-g^{j p} g^{q s} S_{p q}^{t} S_{t s}^{k} \\
& =\frac{1}{2}\left(C^{j p q} S_{p q}^{k}-C^{k p q} S_{p q}^{j}\right) ;
\end{aligned}
$$

hence

$$
H^{j k}-H^{k j}=C_{; i}^{i k j}+F^{j k}+\frac{1}{2}\left(C^{j p q} S_{p q}^{k}-C^{k p q} S_{p q}^{j}\right) .
$$

We calculate the covariant derivative

$$
C_{; i}^{i k j}=-C_{; i}^{i j k}=-\left(C_{, i}^{i j k}+\Gamma_{p i}^{j} C^{i p k}+\Gamma_{p i}^{k} C^{i j p}+\Gamma_{p i}^{i} C^{p k j}\right) .
$$

By virtue of the fact that tensor $C^{i j k}=g^{p j} g^{q k} S_{p q}^{i}+$ $g^{p k} g^{q i} S_{p q}^{j}+g^{p i} g^{q j} S_{p q}^{k}$ is antisymmetric, we have

$$
\begin{aligned}
\Gamma_{p i}^{j} C^{i p k} & =\Gamma_{i p}^{j} C^{i p k}=\frac{1}{2}\left(\Gamma_{i p}^{j} C^{i p k}+\Gamma_{p i}^{j} C^{p i k}\right)=\frac{1}{2} S_{i p}^{j} C^{p k i} \\
& =-\frac{1}{2} S_{p i}^{j} C^{k p i} ;
\end{aligned}
$$

similarly, we obtain

$$
\Gamma_{p i}^{k} C^{i j p}=\Gamma_{i p}^{k} C^{p j i}=\frac{1}{2} C^{j p i}\left(\Gamma_{i p}^{k}-\Gamma_{p i}^{k}\right)=\frac{1}{2} S_{i p}^{k} C^{j p i}=\frac{1}{2} S_{p q}^{k} C^{j q p} .
$$

Then we write,

$$
\begin{aligned}
C_{; i}^{i k j}= & -C_{; i}^{i j k} \\
= & -C_{, i}^{i j k}-\frac{1}{2} S_{p q}^{j} C^{k p q}+\frac{1}{2} S_{p q}^{k} C^{j p q} \\
& -\Gamma_{p q}^{q} C^{p k j}, \\
H^{j k}-H^{k j}= & -C_{, i}^{i j k}+\frac{1}{2} S_{p q}^{j} C^{k p q}-\frac{1}{2} S_{p q}^{k} C^{j p q} \\
& -\Gamma_{p q}^{q} C^{p k j}+F^{j k}+\frac{1}{2}\left(C^{j p q} S_{p q}^{k}-C^{k p q} S_{p q}^{j}\right), \\
H^{j k}-H^{k j}= & -C_{, i}^{i j k}-\Gamma_{p q}^{q} C^{p k j}+F^{j k} .
\end{aligned}
$$

We will compute $\Gamma_{l p}^{p}$; for this, we recall that $\Gamma_{p l}^{p}=$ $(1 / 2) g_{i p, l} g^{i p}=(1 / \sqrt{g})\left(\partial \sqrt{g} / \partial x^{l}\right)$, and $\Gamma_{l p}^{p}=\Gamma_{p l}^{p}+S_{l p}^{p}$ and obtain

$$
\Gamma_{p l}^{p}=\frac{1}{\sqrt{-g}} \frac{\partial \sqrt{-g}}{\partial x^{l}}+(\ln \psi)_{, l}=(\ln (\psi \sqrt{-g}))_{, l} .
$$

Then we obtain

$$
H^{j k}-H^{k j}-F^{j k}=-C_{, i}^{i j k}-(\ln (\psi \sqrt{-g}))_{, i} C^{i k j} .
$$

We multiply by $\psi \sqrt{-g}$ and have

$$
\begin{aligned}
\psi & \sqrt{-g}\left(H^{j k}-H^{k j}-F^{j k}\right) \\
& =-\psi \sqrt{-g}\left(C_{, i}^{i j k}+(\ln (\psi \sqrt{-g}))_{, i} C^{i k j}\right), \\
\psi & \sqrt{-g}\left(H^{j k}-H^{k j}-F^{j k}\right) \\
& =-\left(\psi \sqrt{-g} C^{i j k}\right)_{, i} .
\end{aligned}
$$

We differentiate the last equality, in view of the antisymmetry of the tensors, and we obtain the next important equality:

$$
\left(\psi \sqrt{-g}\left(H^{j k}-H^{k j}-F^{j k}\right)\right)_{, k}=0 .
$$

\section{The Field Equations}

Below we consider the derivation of the field equations in depending on conditions.

6.1. The Field Equations in the Absence of Symmetry Conditions. Let $\Gamma_{j k}^{i}$ be constructed on the basis of connection $n$-dimensional manifold. It is not assumed that the $\Gamma_{j k}^{i}$ are symmetric in $j$ and $k$. Regardless of connection $\Gamma_{j k}^{i}$, we introduce symmetric metric tensor $g_{i k}(4)$.

We will derive the field equations from the variation principle of least action, by varying the function $\Gamma_{j k}^{i}$ and $g_{i k}$ independently.

Using (23) we can write the Riemann tensor

$$
R_{i j}=\Gamma_{i p, j}^{p}-\Gamma_{i j, p}^{p}+\Gamma_{q j}^{p} \Gamma_{i p}^{q}-\Gamma_{q p}^{p} \Gamma_{i j}^{q} .
$$

We form the scalar density as $\left(R_{i k}+S_{i l}^{j} S_{k j}^{l}\right) g^{i k} \sqrt{-g}$, where $S_{j k}^{i}=$ $\Gamma_{j k}^{i}-\Gamma_{k j}^{i}$, and postulate that all the variations of the integral

$$
\int\left(R_{i k}+S_{i l}^{j} S_{k j}^{l}\right) g^{i k} \sqrt{-g} d V
$$

with respect to $\Gamma_{j k}^{i}$ and $g^{i k} \sqrt{-g}$ as the independent variables, are zero (at the boundaries they do not vary).

Without dwelling on the standard intermediate calculations, we find that the variation with respect to $g^{i k} \sqrt{-g}$ leads to the equation

$$
R_{i k}+S_{i l}^{j} S_{k j}^{l}=0
$$

then the variation with respect to $\Gamma_{j k}^{i}$ gives the equation

$$
\begin{aligned}
g_{, k}^{i j}- & \frac{1}{2} g^{i j} g_{p q} g_{, k}^{p q}+g^{p j} \Gamma_{p k}^{i}+g^{i p} \Gamma_{k p}^{j} \\
& -\delta_{k}^{j}\left(g_{, p}^{i p}-\frac{1}{2} g^{i p} g_{m n} g_{, p}^{m n}+g^{p q} \Gamma_{p q}^{i}\right)-g^{i j} \Gamma_{k p}^{p} \\
& +2\left(g^{i p} S_{p k}^{j}+g^{p j} S_{k p}^{i}\right)=0 .
\end{aligned}
$$


If we contract the left side of the last equality by indices $i$ and $k$, then we obtain zero identically equal to zero. If we contract by indices $j$ and $k$, we obtain the equation

$$
-3 g_{, j}^{i j}+\frac{3}{2} g^{i j} g_{p q} g_{, j}^{p q}-3 g^{p q} \Gamma_{p q}^{i}+g^{i p} S_{p j}^{j}=0
$$

Therefore, we have

$$
\begin{aligned}
g_{, k}^{i j}- & \frac{1}{2} g^{i j} g_{p q} g_{, k}^{p q}+g^{p j} \Gamma_{p k}^{i}+g^{i p} \Gamma_{k p}^{j}-g^{i j} \Gamma_{k p}^{p} \\
& -\frac{1}{3} \delta_{k}^{j} g^{i p} S_{p q}^{q}+2\left(g^{i p} S_{p k}^{j}+g^{p j} S_{k p}^{i}\right)=0 .
\end{aligned}
$$

Then we lowered upper indices by using the metric and obtain the equation

$$
\begin{gathered}
-g_{i j, k}-\frac{1}{2} g_{i j} g^{p q} g_{p q, k}+g_{i p} \Gamma_{k j}^{p}+g_{j p} \Gamma_{i k}^{p}-g_{i j} \Gamma_{k p}^{p} \\
-\frac{1}{3} g_{j k} S_{i p}^{p}+2\left(g_{p j} S_{i k}^{p}+g_{i p} S_{k j}^{p}\right)=0 .
\end{gathered}
$$

We use the symmetry of tensor $g_{i j}$ and rearrange $i$ and $j$ and deduce the equation

$$
5\left(g_{i p} S_{k j}^{p}+g_{j p} S_{i k}^{p}\right)+\frac{1}{3}\left(g_{j k} S_{i p}^{p}+g_{i k} S_{j p}^{p}\right)=0 .
$$

Further, we obtained

$$
\begin{gathered}
-g_{i j, k}-\frac{1}{2} g_{i j} g^{p q} g_{p q, k}+g_{i p} \Gamma_{k j}^{p}+g_{j p} \Gamma_{i k}^{p}-g_{i j} \Gamma_{k p}^{p} \\
-\frac{1}{3} g_{j k} S_{i p}^{p}-\frac{2}{15}\left(g_{k j} S_{i p}^{p}+g_{i k} S_{j p}^{p}\right)=0 .
\end{gathered}
$$

Thus, we obtained the field equations (122) by varying connection obtained equation and as a result of them we obtained (123) where there are only metric and torsion. We assuming that the electromagnetic component is absent $\varphi \equiv$ 0 , then, from (123) we have $S_{j k}^{i} \equiv 0$, and connection is symmetrical, as in Riemann geometry and (124) shows the known law of Einstein-Hilbert problem for the gravitational field. If $\varphi \neq 0$ and the metric is flat (no gravitational field), then from (124) can be obtained Maxwell equations for electromagnetic field in vacuum.

6.2. The Einstein-Hilbert Equation in Case of the Absence of Symmetry Conditions, When the Lagrange Function Is Depending on the Torsion Tensor. Now we also start from the variation principle of the least action in the form $\delta\left(W_{m}+\right.$ $\left.W_{g}\right)=0$, where $W_{m}$ and $W_{g}$-action, respectively, for matter and field values, we are varying $g_{i k}$.

By standard calculations, we have

$$
\begin{gathered}
\delta \int\left(R_{i k}+S_{i l}^{j} S_{k j}^{l}\right) g^{i k} \sqrt{-g} d V \\
=\int\left(R_{i k} \sqrt{-g} \delta g^{i k}+R_{i k} g^{i k} \delta \sqrt{-g}+g^{i k} \sqrt{-g} \delta R_{i k}\right. \\
\left.+S_{i l}^{j} S_{k j}^{l} \sqrt{-g} \delta g^{i k}+S_{i l}^{j} S_{k j}^{l} g^{i k} \delta \sqrt{-g}\right) d V, \\
R_{i k} g^{i k} \delta \sqrt{-g}=-\frac{1}{2} R_{p q} g^{p q} g_{i k} \sqrt{-g} \delta g^{i k} .
\end{gathered}
$$

Similarly, we obtain

$$
S_{i l}^{j} S_{k j}^{l} g^{i k} \delta \sqrt{-g}=-\frac{1}{2} S_{p l}^{j} S_{k q}^{l} g^{p q} g_{i k} \sqrt{-g} \delta g^{i k}
$$

Now we compute $g^{i k} \sqrt{-g} \delta R_{i k}$ directly by using the definition and thus obtain two types of summands, the first has the standard form $g^{i k}\left(\delta \Gamma_{k i}^{l}\right)_{, l}-g^{i k}\left(\delta \Gamma_{k l}^{l}\right)_{, i}=\left(g^{i k} \delta \Gamma_{k i}^{l}-g^{i l} \delta \Gamma_{l p}^{p}\right)_{,}$, where it is considered that $g_{, l}^{i k}=0$ and by Stokes' theorem turns into zeros. The term of the second type exists due to the absence of symmetry connection: $g^{i k} \delta R_{i k}=g^{i k} \delta\left(\Gamma_{q p}^{p} \Gamma_{k i}^{q}-\right.$ $\left.\Gamma_{q i}^{p} \Gamma_{k p}^{q l}\right)$. Then we express the connection coefficients via the metric and torsion, and after a rather lengthy calculation, we obtain

$$
g^{i k} \delta R_{i k}=\left(S_{i p}^{p} S_{q k}^{q}-S_{i q}^{p} S_{p k}^{q}-g_{k p} g^{q t} S_{i t}^{m} S_{q m}^{p}\right) \delta g^{i k}
$$

Thus, we have

$$
\begin{gathered}
\delta \int\left(R_{i k}+S_{i l}^{j} S_{k j}^{l}\right) g^{i k} \sqrt{-g} d V \\
=\int\left(R_{i k}-\frac{1}{2} g_{i k} R+\left(S_{i p}^{p} S_{q k}^{q}+S_{i p}^{q} S_{k q}^{p}+g_{k p} g^{q t} S_{i t}^{m} S_{m q}^{p}\right)\right. \\
\left.+S_{i l}^{j} S_{k j}^{l}-\frac{1}{2} S_{p l}^{j} S_{k q}^{l} g^{p q} g_{i k}\right) \sqrt{-g} \delta g^{i k} d V .
\end{gathered}
$$

Then we obtain the conclusions

$$
\begin{aligned}
& \delta W_{g} \\
& =K_{1} \int\left(R_{i k}+S_{i l}^{j} S_{k j}^{l}\right) g^{i k} \sqrt{-g} d V \\
& =K_{1} \int\left(R_{i k}-\frac{1}{2} g_{i k} R+\left(S_{i p}^{p} S_{q k}^{q}+S_{i p}^{q} S_{k q}^{p}+g_{k p} g^{q t} S_{i t}^{m} S_{m q}^{p}\right)\right. \\
& \left.\quad+S_{i l}^{j} S_{k j}^{l}-\frac{1}{2} S_{p l}^{j} S_{k q}^{l} g^{p q} g_{i k}\right) \sqrt{-g} \delta g^{i k} d V,
\end{aligned}
$$

where a physical constant $K_{1}$, as a rule, in a classic case is $c^{3} / 16 \pi k$ and $k$ is called the universal gravitational constant.

For variation of the action of matter, we find

$$
\delta W_{g}=K_{2} \int T_{i k} \sqrt{-g} \delta g^{i k} d V
$$

where $T_{i k}$ is energy-momentum tensor of matter. $K_{2}$ usually take a constant equal to $-1 / 2 c$.

Therefore, by the principle of least action for $\delta W_{g}+\delta W_{m}=$ 0 , we find relations

$$
\begin{aligned}
& \int\left(R_{i k}-\frac{1}{2} g_{i k} R+\left(S_{i p}^{p} S_{q k}^{q}+S_{i p}^{q} S_{k q}^{p}+g_{k p} g^{q t} S_{i t}^{m} S_{m q}^{p}\right)\right. \\
& \left.\quad+S_{i l}^{j} S_{k j}^{l}-\frac{1}{2} S_{p l}^{j} S_{k q}^{l} g^{p q} g_{i k}-K T_{i k}\right) \sqrt{-g} \delta g^{i k} d V=0,
\end{aligned}
$$


because of the arbitrariness $\delta g^{i k}$, we have

$$
\begin{aligned}
R_{i k}- & \frac{1}{2} g_{i k} R+S_{i p}^{p} S_{q k}^{q}+S_{i p}^{q} S_{k q}^{p}+g_{k p} g^{q t} S_{i t}^{m} S_{m q}^{p}+S_{i l}^{j} S_{k j}^{l} \\
& -\frac{1}{2} S_{p l}^{j} S_{k q}^{l} g^{p q} g_{i k}=K T_{i k},
\end{aligned}
$$

where the constant $K$ can be determined by $K_{1}$ and $K_{2}$.

\section{Conclusions}

We have investigated the properties of the space generated jointly and agreed on by the metric and the torsion tensors. We have presented the structure of the curvature tensor and studied its special features and for this tensor we obtained analog Ricci-Jacobi identity and also evaluated the gap that occurs at the transition from the original to the image and vice versa, in the case of an infinitely small contours. The geodesic lines equation has been researched. We have shown that the structure of tensor $\pi_{\alpha \beta}$, which is similar to the second fundamental tensor of hypersurfaces $Y^{n-1}$, is substantially different from the case of Riemannian spaces with zero torsion. Then we have obtained formulas for hypersurfaces $Y^{n-1}$, which characterize the change of vectors in accompanying basis relative to this basis itself.

Taking into consideration the structure of the space with metric and torsion we have reach the aim of our paper that is derived from the variation principle the general fields equations (electromagnetic and gravitational) so we are obtained analog of Einstein-Hilbert equation in space $Y^{n}$.

\section{Conflict of Interests}

The author declares that there is no conflict of interests regarding the publication of this paper.

\section{References}

[1] K. Bredies, "Symmetric tensor fields of bounded deformation," Annali di Matematica Pura ed Applicata, vol. 192, no. 5, pp. 815851, 2013.

[2] E. Cartan and J. Schouten, "On Riemannian geometries admitting an absolute parallelism," Nederlandse Akademie van Wetenschappen. Proceedings Series A, vol. 29, pp. 933-946, 1926.

[3] I. Agricola and T. Friedrich, "On the holonomy of connections with skew-symmetric torsion," Mathematische Annalen, vol. 328, no. 4, pp. 711-748, 2004.

[4] I. Agricola and T. Friedrich, "A note on flat metric connections with antisymmetric torsion," Differential Geometry and Its Applications, vol. 28, no. 4, pp. 480-487, 2010.

[5] B. Alexandrov and S. Ivanov, "Vanishing theorems on Hermitian manifolds," Differential Geometry and its Applications, vol. 14, no. 3, pp. 251-265, 2001.

[6] A. Saa, "A geometrical action for dilaton gravity," Classical and Quantum Gravity, vol. 12, no. 8, pp. L85-L88, 1995.

[7] G. Bonneau, "Compact Einstein-Weyl four-dimensional manifolds," Classical and Quantum Gravity, vol. 16, no. 3, pp. 10571068, 1999.
[8] E. Cartan and J. Schouten, "On the geometry of the group manifold of simple and semisim-ple groups," Nederlandse Akademie van Wetenschappen. Series A, vol. 29, pp. 803-815, 1926.

[9] G. R. Cavalcanti, "Reduction of metric structures on Courant algebroids," The Journal of Symplectic Geometry, vol. 4, no. 3, pp. 317-343, 2006.

[10] T. Dereli and R. W. Tucker, "An Einstein-Hilbert action for axi-dilaton gravity in four dimensions," Classical and Quantum Gravity, vol. 12, no. 4, pp. L31-L36, 1995.

[11] A. Einstein, The Meaning of Relativity, Princeton University Press, Princeton, NJ, USA, 1921.

[12] A. Einstein, Relativity: The Special and General the Ory, H. Holt and Company, New York, NY, USA, 1920.

[13] A. Einstein, "Theorie unitaire de champ physique," Annales de l'Institut Henri Poincaré, no. 1, pp. 1-24, 1930.

[14] S. Manoff, "Frames of reference in spaces with affine connections and metrics," Classical and Quantum Gravity, vol. 18, no. 6, pp. 1111-1125, 2001.

[15] R. A. Mosna and A. Saa, "Volume elements and torsion," Journal of Mathematical Physics, vol. 46, no. 11, Article ID 112502, 2005.

[16] J. A. Peacock, Cosmological Physics, Cambridge University Press, Cambridge, UK, 1999.

[17] P. J. E. Peebles, Principles of Physical Cosmology, Princeton University Press, Princeton, NJ, USA, 1993.

[18] W. Rindler, Essential Relativity. Special, General and Cosmological, Texts and Monographs in Physics, Springer, New York, NY, USA, 2nd edition, 1977.

[19] J. Jost, Riemannian Geometry and Geometric Analysis, Springer, Berlin, Germany, 2005.

[20] H. Pedersen and A. Swann, "Riemannian submersions, fourmanifolds and Einstein-Weyl geometry," Proceedings of the London Mathematical Society. Third Series, vol. 66, no. 2, pp. 381399, 1993.

[21] S. Dineen, Multivariate Calculus and Geometry, Springer Undergraduate Mathematics Series, Springer, Berlin, Germany, 3rd edition, 2014.

[22] J. G. Vargas, Differential Geometry for Physicists and Mathematicians: Moving Frames and Differential Forms: From Euclid Past Riemann, World Scientific, 2014. 

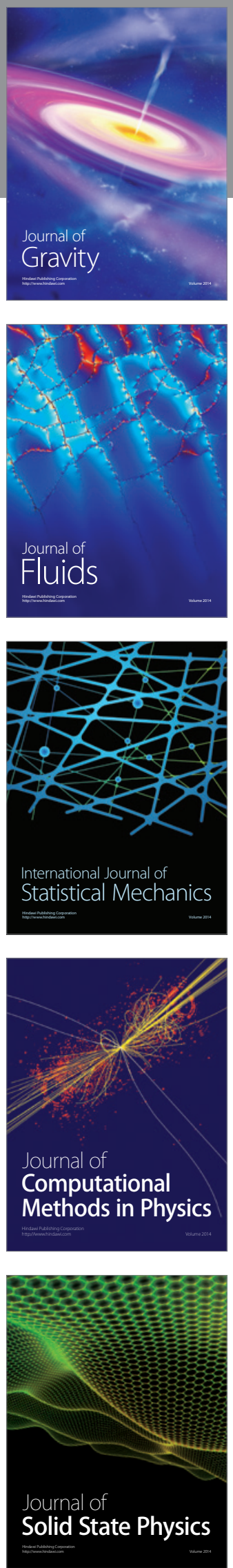

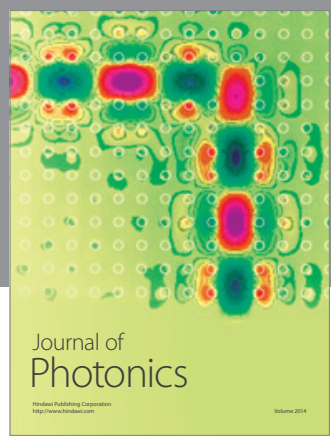

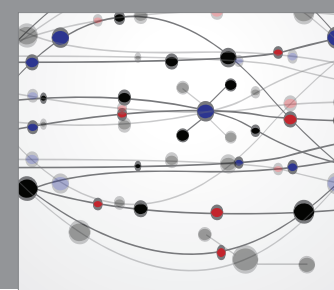

The Scientific World Journal

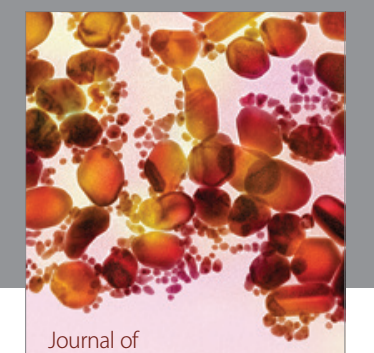

Soft Matter
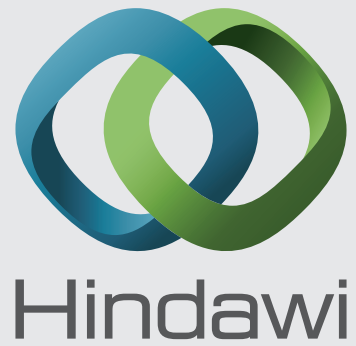

Submit your manuscripts at

http://www.hindawi.com
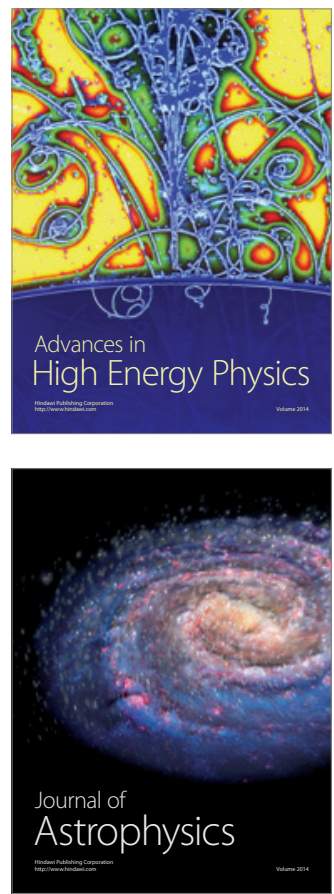
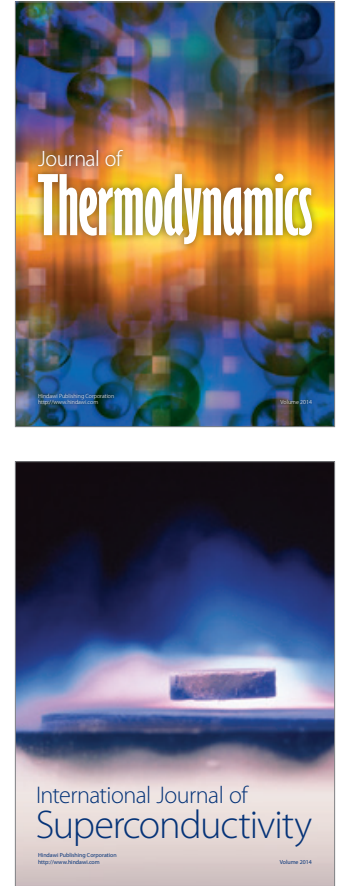
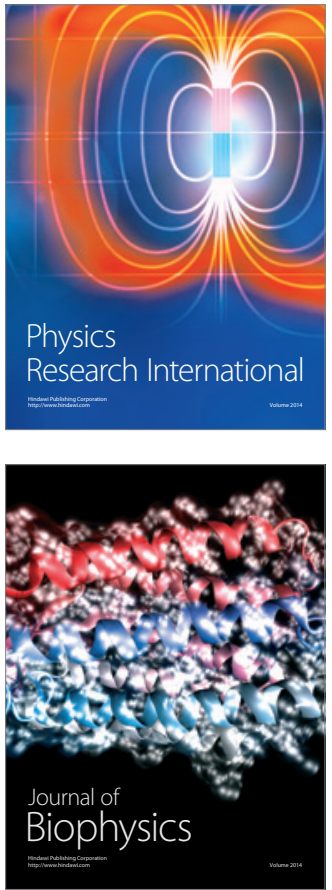
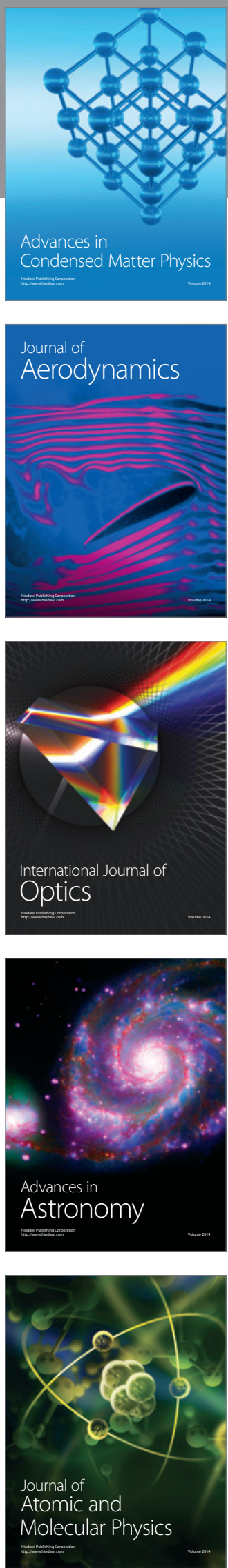